Case Report

\title{
A Case of Hypertension with Systemic Multiple Arterial Stenosis Accompanied by Acute Cerebellar Infarction
}

\author{
Yun Pan ${ }^{1}$, Hailing Liao", Dongdong Chen ${ }^{1, ~ *, ~ J i a x i o n g ~ T a n ~}{ }^{2, *}$ \\ ${ }^{1}$ Department of Cardiology, the First Affiliated Hospital of Jinan University, Guangzhou, China \\ ${ }^{2}$ Department of Hematology, the First Affiliated Hospital of Jinan University, Guangzhou, China \\ Email address: \\ lajiao_420@163.com (Dongdong Chen),gdydtjx@163.com (Jiaxiong Tan) \\ ${ }^{*}$ Corresponding author
}

\section{To cite this article:}

Yun Pan, Hailing Liao, Dongdong Chen, Jiaxiong Tan. A Case of Hypertension with Systemic Multiple Arterial Stenosis Accompanied by Acute Cerebellar Infarction. World Journal of Medical Case Reports. Vol. 9, No. 3, 2020, pp. 54-57. doi: 10.11648/j.sjcm.20200903.13

Received: August 19, 2020; Accepted: August 27, 2020; Published: September 14, 2020

\begin{abstract}
Hypertension is a recognized risk factor for stroke and recurrent stroke. Studies have found that more than $75 \%$ of patients with acute stroke are associated with elevated blood pressure. We report a patient of hypertension with ischemic stroke who had a history of lymphoma and developed systemic atherosclerosis with good control of long-term blood glucose and lipid. Based on the literature, the etiology and treatment were discussed, suggesting that the cause of atherosclerosis may be related to the radiotherapy, chemotherapy and targeted therapy of lymphoma. It is pointed out that the target of blood pressure should be determined according to the actual situation of the patient rather than guadlines only. At the same time, the difference between stroke and coronary arterial disease in the secondary prevention was also revealed. Ticagrelor does not reduce the risk in the secondary prevention of ischemic stroke, and 21-day dual antiplatelet therapy (aspirin and clopidogrel) is recommended for patients with mild stroke starting within 24 hours to prevent stroke recurrence within 90 days.
\end{abstract}

Keywords: Hypertension, Ischemic Stroke, Atherosclerosis, Arterial Stenosis, The Secondary Prevention of Ischemic Stroke

\section{Introduction}

Hypertension is common in stroke, and studies have found that more than $75 \%$ of patients with stroke are associated with elevated blood pressure [1,2]. Although many previous studies have demonstrated that effective regulation of blood pressure in the primary and secondary prevention of stroke can significantly reduce the incidence and recurrence rate of stroke $[3,4]$. However, there is no clear recommendation on the choice of antihypertensive drugs and the target of blood pressure in the current guidelines. We report a patient of hypertension with ischemic stroke who had a history of lymphoma and developed systemic atherosclerosis. And then discussed the etiology and treatment based on the literature. It is believed that radiotherapy, chemotherapy and targeted therapy for lymphoma can also cause cardiac toxicity and atherosclerosis. In addition, the target of blood pressure in treatment should not only refer to the guidelines, but also be determined according to the tolerance of the individual.

\section{Case Report}

The patient was a 71-year-old man who was admitted to hospital mainly due to "intermittent chest tightness for 14 years, repeated dizziness for 1 week". Fourteen years ago, an intermittent chest tightness was happened with no incentive, which can be relieved by rest without treatment. 2016 was the first time this patient has been received coronary angiography owing to the aggravation of chest tightness: the stenosis of left anterior descending (LAD), left circumflex (LCX) and right coronary artery (RCA) was about $80-85 \%, 80-85 \%$ and $80-90 \%$ respectively, and two stents were implanted into LAD and LCX. The symptoms were relieved after the treatment. And after the PCI, aspirin $100 \mathrm{mg} / \mathrm{QD}$ and clopidogrel $75 \mathrm{mg} / \mathrm{QD}$ were taken orally regularly, and aspirin $100 \mathrm{mg} / \mathrm{QD}$ was maintained as a single drug after 18 months. One week ago, the patient had dizziness at rest, which was non-vertigo, accompanied by sweating, headache, nausea, no vomiting, no 
disturbance of consciousness, no blurred vision, no limb weakness, no convulsion, etc. The above symptoms lasted for 5-10 minutes at a time and can be relieved without treatment, but still occurred repeatedly. 10 years ago he was diagnosed with hypertension, the blood pressure was not well controlled due to irregular treatment. Diagnosed with diabetes over 20 years, luckily the blood glucose was controlled very stable because of the regular insulin injection; Three years ago, diffuse large B-cell lymphoma, STAGE IIIA non-GCB, was diagnosed due to the presence of a right neck mass with fever. Four times of R-CDOP (Rituximab, cyclophosphamide, liposome doxorubicin, vincristine, glucocorticoid), one time of R-EPOCH (Rituximab, etoposide (VP-16), liposome doxorubicin, vincristine), five times of R-GEMOX (Rituximab, gemcitabine, oxaliplatin) chemotherapy were administered. After 31 times of local radiotherapy to lymph nodes in the inguinal region, lymphoma was completely relieved and then treated with immunomodulator and the angiogenesis inhibitor Lenalidomide for 2 years. The condition of lymphoma was stable. The patient denied a history of smoking. But his father, siblings also had a history of high blood pressure. Physical examination: Height: $165.5 \mathrm{~cm}$, weight: $70.6 \mathrm{~kg}$, BMI: 25.8 , heart rate: $78 \mathrm{bpm}$, blood pressure: 191/83mmhg (right upper limb), 124/72mmhg (left upper limb). Cardiac rhythm was normal, aortic valve area can be heard diastolic murmurs. The neurological examination was negative. Related blood biochemistry test are shown below: low density lipoprotein cholesterol (LDL-C)

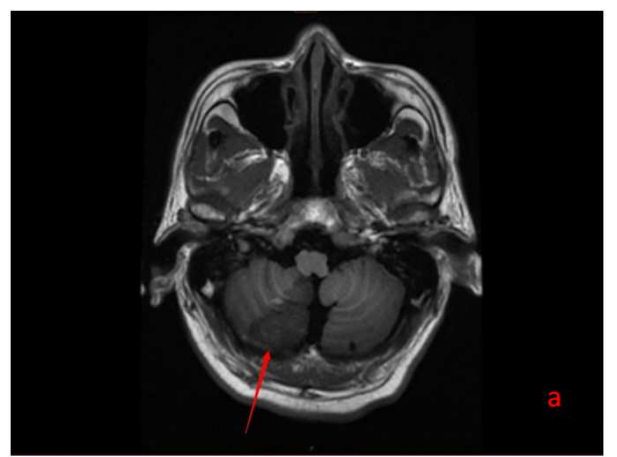

a: T1

$1.06 \mathrm{mmol} / \mathrm{L}$, Total cholesterol (TCHOL) $2.5 \mathrm{mmol} / \mathrm{L}$, Total cholesterol (TG) $1.04 \mathrm{mmol} / \mathrm{L}$, high density lipoprotein cholesterol (HDL-C) $0.65 \mathrm{mmol} / \mathrm{L}$, Homocysteine (HCY) 6umol/L. Hemoglobin A1C: $6.0 \%$. No abnormalities were found in coagulation function, D-dimer, troponin, pro-BNP, tumor markers, thyroid function, rheumatism, ENA lineage, and ANCA vasculitis associated antibodies. A magnetic resonance imaging (MRI) scan (Figure 1) showed acute cerebral infarction in the right cerebellar hemisphere and vermis. Cervical vascular ultrasound indicated the initial stenosis of the right internal carotid artery, with a stenosis degree of about $70 \%$, and bilateral vertebral artery occlusion was considered. And the CT scan showed severe stenosis of the left subclavian artery (Figure 2). The clinical diagnoses were considered as following: 1 . acute ischemic stroke (right cerebellar hemisphere and vermis) 2. Arteriosclerotic cardiovascular disease, unstable angina Post-PCI NYHA class II 3. Bilateral vertebral artery occlusion 4. Systemic stenosis (left subclavian artery severe stenosis) 5. Level 3 hypertension (very high risk) 6. Diabetes 7. Diffuse large B-cell lymphoma, stage III, group A, non-GCB type (complete remission). During the hospital, Ticagrelor was given $90 \mathrm{mg}$ bid for antiplatelet, blood pressure was controlled by $240 \mathrm{mg}$ Alisartan, 5mg Bisoprol, 30mg Nifedipine, 20mg Furosemide and $20 \mathrm{mg}$ spironolactone for day. Rosuvastatin $10 \mathrm{mg} / \mathrm{QN}$ was given for lipid control. In the end, Uniolex 50 was given $28 \mathrm{U}$ /IH before breakfast and $18 \mathrm{U} / \mathrm{IH}$ before dinner to control blood glucose.

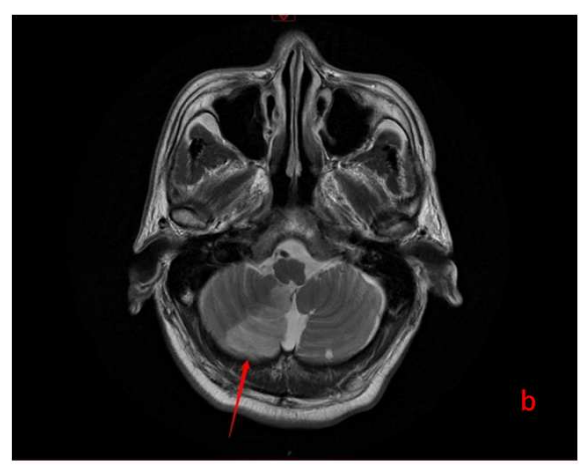

b: T2

Figure 1. Magnetic resonance imaging (MRI) of skull.
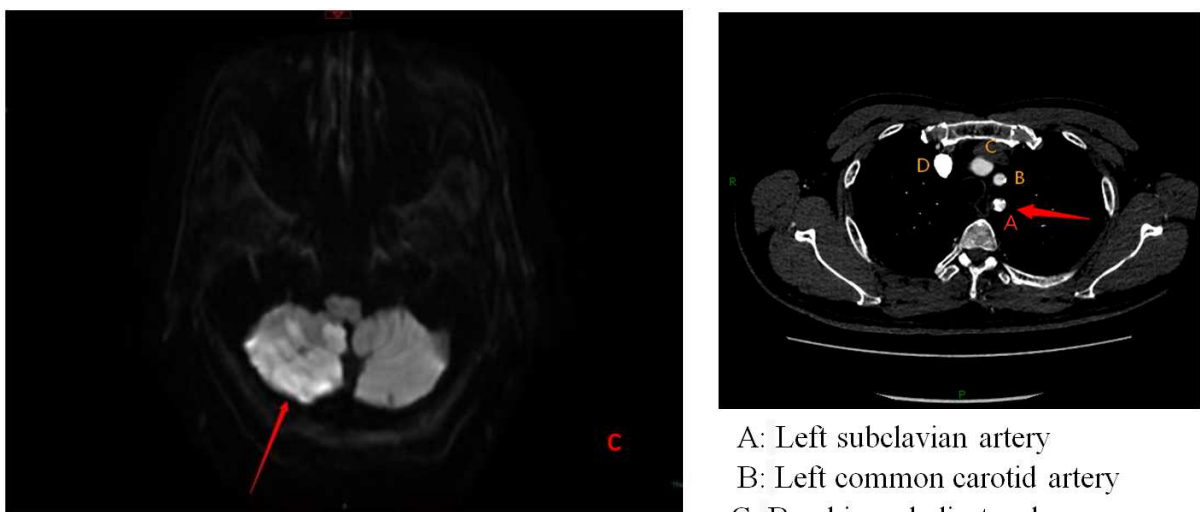

A: Left subclavian artery

B: Left common carotid artery

C: Brachiocephalic trunk

c: DWI

D: Right subclavian artery

Figure 2. Computed tomography (CT) of chest. 


\section{Discussion}

According to the 2018 AHA guidelines, this patient is classified as a very high risk ASCVD population with a target of LDL-C less than $1.4 \mathrm{mmol} / \mathrm{L}$ or a reduction of more than $50 \%$ from baseline. The LDL-C of the patient was only $1.06 \mathrm{mmol} / \mathrm{L}$ when he first entered the hospital, and the HbA1c was $6.0 \%$. It is a difficult problem in the etiological diagnosis of this case that systemic arterial disease develops under the condition of good long-term control of blood glucose and lipid. Atherosclerosis is a common vascular pathological change, can involve arteries throughout the body and is an important pathological basis for the occurrence and progression of a series of disabling and fatal diseases (including coronary arterial disease, stroke and peripheral vascular lesions). Current studies have shown that the common risk factors of atherosclerosis are hypertension, smoking and metabolic syndrome [5]. However, the above data showed that the patient had good long-term control of blood glucose and lipid, just poor blood pressure control alone did not seem to fully explain systemic multi-atherosclerosis and severe stenosis. In addition, a few uncommon causes such as mycoplasma pneumoniae infection, radiotherapy, chemotherapy, and targeted therapy have also been reported to cause cardiotoxicity and lead to atherosclerosis $[6,7]$. The patient was diagnosed with lymphoma 3 years ago, it is not clear whether Rituximab, a drug used during chemotherapy, or Lenalidomide taken orally at a later stage is involved. While previous studies have shown that specific B-cell subsets are important regulators of pro-inflammatory and anti-inflammatory effects in atherosclerosis [8, 9]. For example, B-1 cells play a protective role in atherosclerosis, mainly by producing IgM antibodies, which are specific for antioxidant epitopes [10]. In addition, the strong phagocytosis of B-1 cells, the indirect regulation of acquired immunity through antigen presentation, and the secretion of interleukin-10 (IL-10) can also play a protective role on atherosclerosis through paracrine action $[11,12]$. The damage and inactivation of these anti-inflammatory regulators during lymphoma treatment may be one of the important causes of systemic atherosclerosis.

Currently, there are no clear guidelines and recommendations on the choice of antihypertensive drugs and the target of blood pressure in patients with acute ischemic stroke associated with hypertension. High blood pressure is common in acute stroke, and studies have found that more than $75 \%$ of patients with acute stroke have elevated blood pressure [13]. The mechanism may be related to the previous history of hypertension or intracranial hypertension during stroke and sympathetic-adrenal medulla system excitation. Although many previous studies have demonstrated that effective blood pressure management can significantly reduce the incidence and recurrence of stroke in both primary and secondary prevention. However, it has also been suggested that active control of blood pressure may lead to deterioration of nervous system by reducing perfusion in ischemic areas of the brain [14]. There are also some differences in the recommendations of the guidelines, The 2018 American Heart Association (AHA) and American Stroke Association (ASA) stroke guidelines [3] suggest that: When blood pressure $\geq$ $220 / 120 \mathrm{mmHg} \quad(1 \mathrm{mmHg}=0.133 \mathrm{kPa})$, the efficacy of antihypertensive therapy within $42-72 \mathrm{~h}$ after the ischemic stroke is unclear, but it can reduce blood pressure by $15 \%$ within $24 \mathrm{~h}$ after the ischemic stroke. According to the expert consensus of hypertension in China in 2018, patients with hypertension within 24 hours after ischemic stroke should be handled with caution, the symptoms such as stress, anxiety, pain, nausea and vomiting, and elevated intracranial pressure should be dealt with first. If the blood pressure continues to rise, when $\mathrm{SBP} \geq 200 \mathrm{mmHg}$ or $\mathrm{DBP} \geq 110 \mathrm{mmHg}$, or accompanied by severe cardiac dysfunction, aortic dissection, hypertensive encephalopathy, the patient can be treated with antihypertensive therapy. For stroke patients with stable condition, antihypertensive therapy should be initiated when the blood pressure is $\geq 140 / 90 \mathrm{mmhg}$, and the target is $<140 / 90$ $\mathrm{mmHg}$. Combined with the above reference, the theoretical target of blood pressure in this patient is $<140 / 90 \mathrm{mmHg}$. However, the patient developed intolerance when SBP < $150 \mathrm{mmHg}$, manifested as dizziness and headache. On the contrary, the symptoms were relieved when SBP was controlled at $150-160 \mathrm{mmHg}$. In conclusion, we draw the following conclusion: the blood pressure target not only needs reference guidelines, but also needs to be determined comprehensively according to the tolerance of the individual.

In addition, PLATO showed that Ticagrelor was superior to clopidogrel in preventing cardiovascular death, myocardial infarction (MI), and stroke, and did not increase the risk of major bleeding events [15]. It also provides faster, stronger and more stable platelet inhibition than clopidogrel [16]. Therefore, aspirin combined with Ticagrelor is a commonly used in the secondary prevention in most patients with coronary arterial disease. However, according to the results of SOCRATES [17], the efficacy of Ticagrelor in stroke patients is not superior to aspirin, and there is no difference in safety between the two. In line with this finding, A meta-analysis [18] showed that Ticagrelor or Prasugrel on top of aspirin did not reduce the risk of total stroke during a median follow-up of 9.2 months as compared with dual antiplatelet therapy (DAPT) with clopidogrel/aspirin (RR, 1.04; $P=0.67)$. At the same time pointed out that novel ADP receptor inhibitors neither reduced the risk of ischemic stroke nor increased the risk of intracranial hemorrhage. However, there was a trend toward higher risk of intracranial hemorrhage in the Ticagrelor group. The 2018 ASA stroke guidelines [3] for Ischemic stroke recommend DAPT (aspirin and clopidogrel) for 21 days starting within 24 hours for patients with mild stroke to prevent stroke recurrence within 90 days. Based on the above literature, after improving the detection of CYP2C19 gene polymorphism, the antiplatelet drug was adjusted to be aspirin $100 \mathrm{mg} / \mathrm{QD}$ combined with clopidogrel 75mg/QD. However, for patients with abnormal CYP2C19 gene causing clopidogrel resistance, how to choose antiplatelet drugs? It is 
still an important issue to be further discussed.

In addition to hypertension, smoking, metabolic syndrome and other common risk factors for atherosclerosis, there are also some rare factors such as tumor radiotherapy, chemotherapy and targeted therapy, which need more theoretical and experimental confirmation in the future. In clinical practice, blood pressure targets not only need reference guidelines, but also need to be determined comprehensively according to an individual's tolerance. In addition, how to choose an anti-platelet drug for the stroke patients who can not tolerate clopidogrel because of the abnormal CYP2C19 gene, is still an important issue that need to in-depth discussion.

\section{Acknowledgements}

Author Dongdong Chen and Jiaxiong Tan are the cocorresponding authors of this paper in no particular order. Due to they have made equally important contributions to the design, data collation and writing of this paper.

\section{References}

[1] Pistoia F, Sacco S, Degan D, et al. Hypertension and Stroke: Epidemiological Aspects and Clinical Evaluation [J]. High blood pressure \& cardiovascular prevention: the official journal of the Italian Society of Hypertension 2016, 23 (1): 9-18.

[2] Cipolla MJ, Liebeskind DS, Chan SL. The importance of comorbidities in ischemic stroke: Impact of hypertension on the cerebral circulation $[\mathrm{J}]$. Journal of cerebral blood flow and metabolism: official journal of the International Society of Cerebral Blood Flow and Metabolism 2018, 38 (12): 2129-2149.

[3] Powers WJ, Rabinstein AA, Ackerson T, et al. Guidelines for the Early Management of Patients With Acute Ischemic Stroke: 2019 Update to the 2018 Guidelines for the Early Management of Acute Ischemic Stroke: A Guideline for Healthcare Professionals From the American Heart Association/American Stroke Association [J]. Stroke 2019, 50 (12): e344-e418.

[4] Hankey GJ. Secondary stroke prevention [J]. The Lancet Neurology 2014, 13 (2): 178-194.

[5] Libby P, Buring JE, Badimon L, et al. Atherosclerosis [J]. Nature reviews Disease primers 2019, 5 (1): 56.

[6] Min SS, Wierzbicki AS. Radiotherapy, chemotherapy and atherosclerosis $[\mathrm{J}]$. Current opinion in cardiology 2017, 32 (4): 441-447.

[7] Seijkens TTP, Lutgens E. Cardiovascular oncology: exploring the effects of targeted cancer therapies on atherosclerosis [J]. Current opinion in lipidology 2018, 29 (5): 381-388.

[8] Perry HM, Bender TP, McNamara CA. B cell subsets in atherosclerosis [J]. Frontiers in immunology 2012, 3: 373.

[9] Srikakulapu P, McNamara CA. B cells and atherosclerosis [J]. American journal of physiology Heart and circulatory physiology 2017, 312 (5): H1060-h1067.

[10] Upadhye A, Sturek JM, McNamara CA. 2019 Russell Ross Memorial Lecture in Vascular Biology: B Lymphocyte-Mediated Protective Immunity in Atherosclerosis [J]. Arteriosclerosis, thrombosis, and vascular biology 2020, 40 (2): 309-322.

[11] Popi AF. B-1 phagocytes: the myeloid face of B-1 cells [J]. Annals of the New York Academy of Sciences 2015, 1362: 86-97.

[12] Alhakeem SS, Sindhava VJ, McKenna MK, et al. Role of B cell receptor signaling in IL-10 production by normal and malignant B-1 cells [J]. Annals of the New York Academy of Sciences 2015, 1362 (1): 239-249.

[13] Qureshi AI. Acute hypertensive response in patients with stroke: pathophysiology and management [J]. Circulation 2008, 118 (2): 176-187.

[14] Johnston KC, Mayer SA. Blood pressure reduction in ischemic stroke: a two-edged sword? [J]. Neurology 2003, 61 (8): 1030-1031.

[15] Wallentin L, Becker RC, Budaj A, et al. Ticagrelor versus clopidogrel in patients with acute coronary syndromes $[\mathrm{J}]$. The New England journal of medicine 2009, 361 (11): 1045-1057.

[16] Gurbel PA, Bliden KP, Butler K, et al. Randomized double-blind assessment of the ONSET and OFFSET of the antiplatelet effects of ticagrelor versus clopidogrel in patients with stable coronary artery disease: the ONSET/OFFSET study [J]. Circulation 2009, 120 (25): 2577-2585.

[17] Johnston SC, Amarenco P, Albers GW, et al. Ticagrelor versus Aspirin in Acute Stroke or Transient Ischemic Attack [J]. The New England journal of medicine 2016, 375 (1): 35-43.

[18] Gouya G, Arrich J, Wolzt M, et al. Antiplatelet treatment for prevention of cerebrovascular events in patients with vascular diseases: a systematic review and meta-analysis [J]. Stroke 2014, 45 (2): 492-503. 